\title{
HIPERTENSIÓN ARTERIAL Y OBESIDAD EN INDÍGENAS ASHÁNINKAS DE LA REGIÓN JUNÍN, PERÚ
}

\author{
Candice Romero ${ }^{1, a}$, Carol Zavaleta ${ }^{2,5,6, a}$, Lilia Cabrera ${ }^{1, b}$, Robert H. Gilman ${ }^{1,3,4, c}$, J. Jaime Miranda ${ }^{3,5, d}$
}

RESUMEN

Con el objetivo de determinar la prevalencia de hipertensión arterial y obesidad en indígenas asháninkas, con limitado contacto con la cultura occidental, se realizó el 2008 un estudio transversal en cinco comunidades asháninkas de la región Junín en la selva del Perú. Se incluyó pobladores de 35 años a más siendo evaluados 76 sujetos (edad promedio 47,4 años, 52,6\% mujeres), correspondientes al $43,2 \%$ de la población elegible. La prevalencia de hipertensión fue 14,5\% (IC 95\%: 6,4-22,6) y de obesidad según índice de masa corporal 4\% (IC 95\%: 0-8,4), sin diferencias según sexo. No hubo diferencias en niveles de presión arterial por grupos de edad. Comparada con estudios previos en pobladores no indígenas de la selva peruana la prevalencia de hipertensión fue elevada. Sin embargo, la prevalencia de obesidad fue baja. Nuestros hallazgos constituyen un llamado para no desatender la situación de las enfermedades crónicas no transmisibles en las poblaciones indígenas de la Amazonia del Perú.

Palabras clave: Hipertensión; Población indígena; Transición de la salud; Transición nutricional; Obesidad; Enfermedad crónica (fuente: DeCS BIREME).

\section{HIGH BLOOD PRESSURE AND OBESITY IN INDIGENOUS ASHANINKAS OF JUNIN REGION, PERU}

\begin{abstract}
In order to determine the prevalence of high blood pressure and obesity in indigenous Ashaninkas, with limited contact with Western culture, a cross-sectional study was conducted in 2008 in five Ashaninka communities of the Junin region in the jungle of Peru. Individuals aged 35 or older were included. 76 subjects were evaluated (average age 47.4 years old, $52.6 \%$ women) corresponding to $43.2 \%$ of the eligible population. The prevalence of hypertension was $14.5 \%$ (Cl 95\%: 6.4-22.6) and the prevalence of obesity, according to body mass index, was $4 \%$ (Cl 95\%: 0-8.4). No differences were observed in gender or in blood pressure levels by age group. Compared with previous studies in non-indigenous people of the Peruvian jungle, the prevalence of high blood pressure was higher while the prevalence of obesity was lower. Our findings are a call to be aware of the situation of chronic non-communicable diseases in indigenous populations in the Peruvian Amazon.
\end{abstract}

Key words: Hypertension; Indigenous population; Health transition; Nutritional transition; Obesity; Chronic disease (source: MeSH NLM).

\section{INTRODUCCIÓN}

Las transiciones a nivel poblacional, tanto la nutricional como la epidemiológica, no son ajenas al Perú (1). Si bien los agregados nacionales demuestran una prevalencia considerable de enfermedades crónicas en el país (2), dichos estimados no consideran a las comunidades indígenas como unidad de evaluación.
Análisis situacionales en grupos indígenas peruanos indican una mayor prevalencia de condiciones transmisibles ${ }^{(3)}$. Sin embargo, reportes internacionales sugieren que en el mundo más del $50 \%$ de los indígenas de más de 35 años de edad padecen diabetes tipo 2, y se prevé que estas cifras aumenten ${ }^{(4)}$. El proceso de aculturación por el que atraviesan las comunidades indígenas conlleva cambios en los estilos de vida, los

\footnotetext{
Área de Investigación y Desarrollo, A. B. PRISMA, Lima, Perú

Unidad Salud Indígena-Fundación Cayetano Heredia. Lima, Perú.

CRONICAS Centro de Excelencia en Enfermedades Crónicas, Universidad Peruana Cayetano Heredia. Lima, Perú.

Department of International Health, Johns Hopkins Bloomberg School of Public Health. Baltimore, EE. UU.

Facultad de Medicina, Universidad Peruana Cayetano Heredia. Lima, Perú.

Departamento de Geografía, Universidad McGill. Montreal, Canadá.

a Médico cirujano; ${ }^{b}$ enfermera, especialista en Salud Pública; ${ }^{c}$ médico, especialista en enfermedades infecciosas; ${ }^{\mathrm{d}}$ médico, magíster y doctor en Epidemiología. Recibido: 12-10-13 Aprobado: 29-01-14
}

Citar como: Romero C, Zavaleta C, Cabrera L, Gilman RH, Miranda JJ. Hipertensión arterial y obesidad en indígenas asháninkas de la región Junín, Perú. Rev Peru Med Exp Salud Publica. 2014;31(1):78-83. 
que pueden incrementar los factores de riesgo para enfermedades crónicas ${ }^{(5)}$.

En medio de los distintos procesos de cambios y transiciones, resulta importante conocer el perfil de enfermedades crónicas en la población indígena peruana con limitado contacto con la cultura occidental. Este estudio tiene por objetivo determinar la prevalencia de hipertensión arterial y obesidad, en indígenas asháninkas del departamento de Junín en la selva del Perú.

\section{EL ESTUDIO}

Estudio transversal realizado en julio del 2008 en cinco comunidades asháninkas (Charahuaja, Capitiri, Ivotsote, Mayapo y Cushireni) del distrito de río Tambo, provincia de Satipo, región de Junín; en la selva peruana, ubicadas a $260 \mathrm{~m}$ de altitud.

El centro poblado más cercano es Puerto Ocopa, distante a tres horas en bote a motor, y la ciudad más cercana, Satipo, está a dos horas más por carretera (Figura 1). Debido a la distancia, costo de transporte y dependiendo del clima, el contacto de los pobladores de esta zona con la cultura occidental es limitado, excepto para la compra y venta de algunos productos. Las comunidades fueron seleccionadas por estar cerca a la rivera en la cuenca del río Tambo, por tener la mayor población según los censos locales y por mantener sus estilos de vida ancestrales lo cual incluye una fuerte relación con el medioambiente para su subsistencia.

Se incluyó a pobladores de 35 a más años, voluntarios, de ambos sexos, que refirieron vivir permanentemente en las comunidades mencionadas y que estuvieron presentes al momento de la visita. Por las limitaciones logísticas de trabajar en áreas remotas, se priorizó la población mayor de 30 años, basados en la evidencia que la presión arterial empieza a incrementarse a esa edad hasta niveles de prehipertensión ${ }^{(6)}$.

Para el cálculo del denominador poblacional, se utilizó el censo local elaborado por los líderes de cada comunidad. Se excluyeron a las mujeres que reportaron estar embarazadas. La etnicidad, se definió por la presencia de dos apellidos indígenas y por autorreconocimiento.

El cuestionario aplicado incluyó preguntas sobre edad, ocupación, grado de instrucción, consumo de tabaco (por lo menos un cigarrillo en el último mes), alcohol (consumo de bebida alcohólica al menos una vez al mes, incluyendo masato fermentado) y autorreporte de enfermedades cardiovasculares diagnosticadas previamente por un personal de salud. El cuestionario fue aplicado en lenguaje asháninka por promotores de salud indígenas bilingües, previamente capacitados.

La presión arterial fue medida con un tensiómetro digital (Omron M5-i, Omron, Japan) validado para población adulta ${ }^{(7)}$. En una única visita se tomaron tres medidas en posición sentada y en reposo; para el análisis se tomó el promedio de las dos últimas medidas. La talla y peso se midieron con el individuo descalzo y con ropa ligera. La longitud de cintura y cadera se midió tres veces con un centímetro inextensible y se tomó el promedio de las tres medidas. Las mediciones para bioimpedancia eléctrica $(B E)$, se hicieron por triplicado, con el individuo recostado sobre una superficie no conducente con ropa holgada y sin portar objetos metálicos en el cuerpo ${ }^{(8)}$. Se usó el

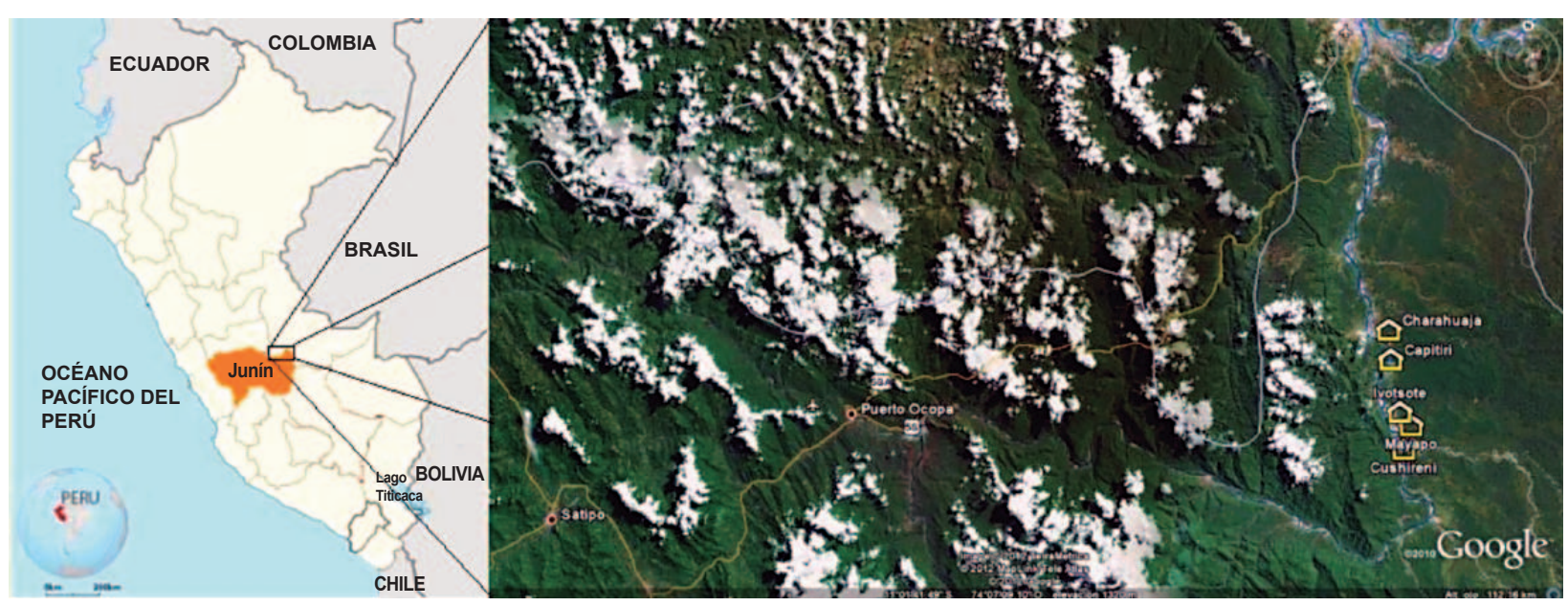

Figura 1. Ubicación de las cinco comunidades asháninkas en la región Junín, Perú 2008 
sistema de BE Quantum II (Clinton Township, MI, USA) y se utilizaron los valores de resistencia y reactancia medidos con el equipo para obtener la impedancia y calcular el porcentaje de grasa corporal con el software proporcionado por el fabricante ${ }^{(8)}$.

La hipertensión arterial fue definida como presión arterial sistólica (PAS) $\geq 140 \mathrm{~mm} \mathrm{Hg}$ o presión arterial diastólica $(P A D) \geq 90 \mathrm{~mm} \mathrm{Hg}$, según estándares internacionales ${ }^{(9)}$.

La obesidad fue definida tanto por índice de masa corporal (IMC) y por BE. Según IMC, se consideró: obesidad (IMC $\geq 30 \mathrm{~kg} / \mathrm{m}^{2}$ ), sobrepeso (IMC $25-29,9 \mathrm{~kg} / \mathrm{m}^{2}$ ), normal (IMC 18,5-24,9 kg/m²) y delgadez (IMC < 18,5 kg/m²) ${ }^{(10)}$. Según BE, obesidad se definió como: $\geq 25 \%$ y $\geq 35 \%$ de grasa corporal en hombres y mujeres, respectivamente (11).

Se calcularon promedios y desviaciones estándar (DE) de las variables numéricas y las proporciones de las variables categóricas a nivel global y por sexo. Los promedios y medianas de las PAS y PAD fueron calculados por cada grupo de edad y se analizaron mediante las pruebas ANOVA y Kruskal-Wallis. Las pruebas exploratorias de asociación fueron realizadas mediante análisis bivariado utilizando la prueba exacta de Fisher. El análisis se realizó con Stata v10.0 (Stata Corporation, College Station, TX).

El protocolo fue aprobado por el comité de ética de la Universidad Peruana Cayetano Heredia y la Asociación Benéfica PRISMA. Se explicaron los objetivos del estudio al jefe y promotores de salud de cada comunidad, quienes, en su lengua, transmitieron la información e invitación a participar a los pobladores presentes. Se obtuvo el consentimiento verbal, y cada voluntario firmó un consentimiento informado escrito.

\section{HALLAZGOS}

De los 928 pobladores en las cinco comunidades asháninkas, $176(18,9 \%$ de la población elegible) fueron mayores de 35 años, de los cuales 76 (43,2\%) participaron en el estudio. El motivo principal de no participación fue estar ausente de la comunidad al momento de la realización del estudio. No hubo diferencias según edad $(p=0,059)$, sexo $(p=0,543)$ y lugar de residencia $(p=0,625)$ entre los sujetos participantes y no participantes en el estudio.

La edad promedio de los participantes fue 47,4 \pm 9,9 años, $52,6 \%$ (40) fueron mujeres y $30,3 \%$ (23) eran analfabetos. Menos del 4\% (3) autorreportó diagnóstico previo de hipertensión arterial y 9\% (7) tuberculosis.
Tabla 1. Descripción sociodemográfica de la población de estudio en cinco comunidades asháninkas del Perú

\begin{tabular}{|c|c|c|c|c|}
\hline & Total & Hombres & Mujeres & $p^{\dagger}$ \\
\hline & $\mathbf{N}(\%)$ & $\mathrm{N}(\%)$ & $\mathbf{N}(\%)$ & $\boldsymbol{p}^{\prime}$ \\
\hline Edad* $^{*}$ & $47,4 \pm 9,9$ & $47,9 \pm 10,3$ & $46,9 \pm 9,6$ & 0,640 \\
\hline Años de estudio* & $3,3 \pm 2,9$ & $4,5 \pm 3,0$ & $2,3 \pm 2,3$ & $<0,001$ \\
\hline \multicolumn{5}{|l|}{ Nivel de educación } \\
\hline Analfabeto & $23(30,3)$ & $6(16,7)$ & $17(42,5)$ & 0,003 \\
\hline Primaria incompleta & $30(39,5)$ & $12(33,3)$ & $18(45,0)$ & \\
\hline Primaria completa & $17(22,4)$ & $13(36,1)$ & $4(10,0)$ & \\
\hline Secundaria incompleta & $6(7,9)$ & $5(13,9)$ & $1(2,5)$ & \\
\hline \multicolumn{5}{|c|}{ Principal actividad laboral } \\
\hline Campesino & $43(56,6)$ & $35(97,2)$ & $8(20,0)$ & $<0,001$ \\
\hline Su casa & $33(43,4)$ & $1(2,8)$ & $32(80,0)$ & \\
\hline \multicolumn{5}{|c|}{ Autorreporte de enfermedad } \\
\hline Hipertensión arterial & $3(3,9)$ & $1(2,8)$ & $2(5,0)$ & 1,000 \\
\hline $\begin{array}{l}\text { Enfermedad cerebro } \\
\text { vascular }\end{array}$ & $1(1,3)$ & 0 & $1(2,5)$ & 1,000 \\
\hline Tuberculosis & $7(9,2)$ & $4(11,1)$ & $3(7,5)$ & 0,702 \\
\hline Asma & $1(1,3)$ & $1(2,8)$ & 0 & 0,474 \\
\hline \multicolumn{5}{|l|}{ Hábitos nocivos } \\
\hline Actualmente fuman $\ddagger$ & $15(19,7)$ & $14(38,9)$ & $1(2,5)$ & $<0,001$ \\
\hline $\begin{array}{l}\text { Consume bebida } \\
\text { alcohólica }^{*}\end{array}$ & $11(14,5)$ & $7(19,4)$ & $4(10,0)$ & 0,243 \\
\hline
\end{tabular}

* Promedio \pm desviación estándar. $\dagger$ Chi-cuadrado. $\ddagger$ por lo menos un cigarrillo en el último mes. $¥$ al menos una vez al mes.

Fumó al menos un cigarrillo en el último mes el 19,7\% (15), y 14,5\% (11) consume bebidas alcohólicas al menos una vez al mes (principalmente una bebida tradicional con cierto grado de alcohol llamada masato) (Tabla 1).

Hubo diferencias significativas entre hombres y mujeres en el promedio de peso, talla, largo de pierna y porcentaje de grasa corporal. No existió diferencia según sexo en otros marcadores vinculados con obesidad, evaluados como perímetros de cintura y cadera, IMC e índice cintura-cadera. Tampoco hubo diferencias significativas en niveles de presión arterial por sexo ni por grupos de edad (Tabla 2).

La prevalencia global de hipertensión arterial fue 14,5\% (IC 95\%: 6,4-22,6). De los once sujetos clasificados como hipertensos el 36,4\% (4) fue de tipo sistodiastólico y el $63,6 \%$ (7) sistólico aislado. Tres sujetos fueron clasificados como obesos por IMC y veinte por BE. La prevalencia global de obesidad según IMC fue 4\% (IC 95\%: 0-8,4), los tres sujetos clasificados como obesos por IMC también lo fueron por BE y todos fueron mujeres. La prevalencia global de obesidad según BE fue 26,3\% (IC 95\%: 16,2-36,4) con una edad promedio de 45,2 años (DE 12,2), mientras que los no obesos tuvieron en promedio 48,2 años (DE 8,9); de los veinte obesos por BE trece fueron mujeres. No hubo 
Tabla 2. Distribución de marcadores antropométricos y presión arterial en cinco comunidades asháninkas del Perú

\begin{tabular}{|c|c|c|c|}
\hline & Hombres & Mujeres & $p^{\ddagger}$ \\
\hline & $\mathrm{N}=36$ & $\mathrm{~N}=\mathbf{4 0}$ & $\rho^{+}$ \\
\hline \multicolumn{4}{|l|}{ Variables antropométricas } \\
\hline Peso $(\mathrm{kg})^{*}$ & $60,5 \pm 8,1$ & $51,9 \pm 8,9$ & $<0,001$ \\
\hline Talla $(\mathrm{cm})^{*}$ & $157,7 \pm 5,1$ & $146,8 \pm 6,3$ & $<0,001$ \\
\hline Largo de pierna $(\mathrm{cm})^{*}$ & $74,5 \pm 3,7$ & $69,2 \pm 4,9$ & $<0,001$ \\
\hline Cintura $(\mathrm{cm})^{\star}$ & $86,1 \pm 5,6$ & $87,6 \pm 9,7$ & 0,401 \\
\hline Cadera $(\mathrm{cm})^{*}$ & $88,9 \pm 4,6$ & $88,5 \pm 6,9$ & 0,718 \\
\hline \multicolumn{4}{|c|}{ Factores de riesgo cardiovascular } \\
\hline Índice de masa corporal $\left(\mathrm{kg} / \mathrm{m}^{2}\right)^{*}$ & $24,2 \pm 2,4$ & $24,1 \pm 3,5$ & 0,814 \\
\hline Índice cintura cadera* & $0,9 \pm 0,04$ & $0,9 \pm 0,06$ & 0,082 \\
\hline Grasa corporal $(\%)^{*}$ & $21,9 \pm 3,5$ & $31,1 \pm 6,7$ & $<0,001$ \\
\hline $\begin{array}{l}\text { Presión arterial sistólica } \\
(\mathrm{mm} \mathrm{Hg})^{*}\end{array}$ & $125,7 \pm 26,2$ & $120,9 \pm 18,5$ & 0,355 \\
\hline $\begin{array}{l}\text { Presión arterial diastólica } \\
(\mathrm{mm} \mathrm{Hg})^{\star}\end{array}$ & $68,9 \pm 10,3$ & $69,5 \pm 9,8$ & 0,792 \\
\hline \multicolumn{4}{|c|}{ Presión arterial según grupos de edad } \\
\hline \multicolumn{4}{|c|}{ Presión arterial sistólica $(\mathrm{mm} \mathrm{Hg})$} \\
\hline $35-44$ & $118,9 \pm 13,8^{*}$ & $117(15,3)^{\dagger}$ & $0,584^{*}$ \\
\hline $45-54$ & $122,7 \pm 17,4^{*}$ & $120(14)^{\dagger}$ & \\
\hline $55-64$ & $137,6 \pm 40,0^{*}$ & $127,8(36,5)^{\dagger}$ & \\
\hline$\geq \operatorname{de} 65$ & $114,9 \pm 7,6^{*}$ & $113,5(10,5)^{\dagger}$ & \\
\hline \multicolumn{4}{|c|}{ Presión arterial diastólica (mm Hg) } \\
\hline $35-44$ & $69,8 \pm 9,5^{*}$ & $69,3(12,8)^{\dagger}$ & $0,164^{*}$ \\
\hline $45-54$ & $71,6 \pm 9,3^{*}$ & $72(10)^{\dagger}$ & \\
\hline $55-64$ & $66,4 \pm 12,5^{*}$ & $64,5(18,5)^{\dagger}$ & \\
\hline$\geq$ de 65 & $63 \pm 5,9^{*}$ & $62,5(3)^{\dagger}$ & \\
\hline
\end{tabular}

* Promedio \pm desviación estándar. $\dagger$ mediana (rango intercuartil).

$\ddagger \mathrm{t}$ de student. ¥ Kruskal-Wallis.

diferencias según sexo en ninguno de estos estimados (Tabla 3).

Ninguno de los factores de riesgo tradicionales evaluados, entre ellos edad, sexo, nivel educativo y marcadores de obesidad, estuvo asociado con hipertensión arterial (Tabla 4).

Tabla 3. Prevalencia de hipertensión arterial y obesidad global en cinco comunidades asháninkas del Perú

\begin{tabular}{|c|c|c|c|c|c|}
\hline & \multicolumn{2}{|r|}{ Total } & \multirow{2}{*}{$\frac{\text { Hombres }}{N(\%)}$} & \multirow{2}{*}{$\frac{\text { Mujeres }}{\mathrm{N}(\%)}$} & \multirow{2}{*}{$p^{*}$} \\
\hline & $\mathbf{N}$ & $\%$ (IC 95\%) & & & \\
\hline $\begin{array}{l}\text { Hipertensión } \\
\text { arterial }\end{array}$ & 11 & $14,5(6,4-22,6)$ & $6(16,7)$ & $5(12,5)$ & 0,747 \\
\hline \multicolumn{6}{|c|}{ Categorías según IMC } \\
\hline Delgadez & 2 & $2,6(0-6,3)$ & 0 & $2(5)$ & 0,233 \\
\hline Normal & 44 & $57,9(46,5-69,3)$ & $23(63,9)$ & $21(52,5)$ & \\
\hline Sobrepeso & 27 & $35,5(24,5-46,5)$ & $13(36,1)$ & $14(35)$ & \\
\hline Obesidad & 3 & $4(0-8,4)$ & 0 & $3(7,5)$ & \\
\hline $\begin{array}{l}\text { Obesidad } \\
\text { por BE }\end{array}$ & 20 & $26,3(16,2-36,4)$ & $7(19,4)$ & $13(32,5)$ & 0,297 \\
\hline
\end{tabular}

Tabla 4. Distribución de hipertensos y no hipertensos en cinco comunidades asháninkas del Perú

\begin{tabular}{|c|c|c|c|c|}
\hline & Total & Hipertensos & $\begin{array}{c}\text { No } \\
\text { hipertensos } \\
\end{array}$ & $\boldsymbol{p}^{\dagger}$ \\
\hline & $\mathbf{N}(\%)$ & N (\%) & $\mathrm{N}(\%)$ & \\
\hline Edad* & $47,4(9,9)$ & $49,8(8,3)$ & $46,9(10,1)$ & $0,379^{\ddagger}$ \\
\hline \multicolumn{5}{|l|}{ Grupo de edad } \\
\hline $35-44$ & $36(47,4)$ & $4(36,4)$ & $32(49,2)$ & 0,131 \\
\hline $45-54$ & $21(27,6)$ & $2(18,2)$ & $19(29,2)$ & \\
\hline $55-64$ & $14(18,4)$ & $5(45,5)$ & $9(13,9)$ & \\
\hline$\geq$ de 65 & $5(6,6)$ & 0 & $5(7,7)$ & \\
\hline Sexo femenino & $40(52,6)$ & $5(45,5)$ & $35(53,9)$ & 0,747 \\
\hline \multicolumn{5}{|l|}{ Nivel de educación } \\
\hline Analfabeto & $23(30,3)$ & $4(36,4)$ & $19(29,2)$ & 0,895 \\
\hline $\begin{array}{l}\text { Primaria } \\
\text { incompleta }\end{array}$ & $30(39,5)$ & $5(45,5)$ & $25(38,5)$ & \\
\hline Primaria completa & $17(22,4)$ & $2(18,2)$ & $15(23,1)$ & \\
\hline $\begin{array}{l}\text { Secundaria } \\
\text { incompleta }\end{array}$ & $6(7,9)$ & 0 & $6(9,2)$ & \\
\hline Sobrepeso* & $30(39,5)$ & $4(36,4)$ & $26(40,0)$ & 1,000 \\
\hline Obesidad & $3(3,9)$ & $1(9,1)$ & $2(3,1)$ & 0,379 \\
\hline Obesidad por BE & $20(26,3)$ & $2(18,2)$ & $18(27,7)$ & 0,717 \\
\hline
\end{tabular}

* Promedio \pm desviación estándar. † prueba exacta de Fisher.

¥ t de Student. ¥ según índice de masa corporal.

BE: bioimpedancia eléctrica

\section{DISCUSIÓN}

Pese a la distancia geográfica con la cultura occidental de la población asháninka estudiada, la prevalencia de hipertensión es similar a la de indígenas que han experimentado grandes cambios sociales tales como migración, incluso es mayor que la de población no indígena de la selva peruana, que mantiene un estilo de vida completamente distinto al de las comunidades nativas ${ }^{(2)}$.

Un estudio que incluyó población urbana y rural de la selva, aunque no de comunidades nativas (2), describe una prevalencia de hipertensión arterial y obesidad por IMC de 11,8 y $15,3 \%$ respectivamente. Los indígenas yanomanis en Brasil (12) o los kunas de Centro América ${ }^{(13)}$, prácticamente no reportan casos de hipertensión. Sin embargo, en indígenas asentados fuera de sus territorios ancestrales, la tasa de hipertensión es de $14,6 \%{ }^{(5)}$. Los kunas ubicados en una zona urbana de Panamá tienen 10,7\% de hipertensión (13).

En Perú se ha reportado cambios en la composición de la dieta en pobladores aguarunas de zonas remotas, sin embargo, los efectos sobre el perfil de salud relacionado a enfermedades crónicas, aún no han sido estudiados ${ }^{(14)}$.

En este estudio hubo un incremento con la edad de los valores promedios de PAS y PAD; aunque esto no fue 
estadísticamente significativo, y en el caso de los mayores de 65 años el promedio resultó menor. Sin embargo, la falta de asociación puede deberse al escaso número de pobladores mayores de 65 años, lo que podría estar vinculado a un probable sesgo de supervivencia.

La prevalencia de obesidad por IMC en los asháninkas participantes es inferior a la de la población no indígena de la Amazonía peruana ${ }^{(2)}$, y a lo reportado en poblaciones indígenas de Brasil quienes enfrentan un rápido cambio socioeconómico y transformaciones ambientales, en quienes la prevalencia de obesidad es de $18^{(15)}$ y $23 \%{ }^{(16)}$. Se ha reportado que los valores culturales, como el de intercambio de alimentos entre parientes y en la comunidad, podrían tener un efecto mitigador en la evolución hacia la obesidad entre pobladores indígenas que han sido expuestos a significativos cambios socioeconómicos (16). En los asháninkas, sería importante investigar la contribución de las características culturales, incluyendo las redes sociales de intercambio de alimentos, la dieta y la actividad física en el desarrollo o mitigación de enfermedades crónicas como obesidad e hipertensión.

Comparado con los promedios nacionales (17), la frecuencia de autorreporte de tuberculosis fue elevada; estudios previos indican que los asháninkas son el pueblo amazónico que más casos de tuberculosis reporta ${ }^{(18)}$. tuberculosis y enfermedades no transmisibles, como hipertensión y obesidad, podrían estar influenciadas por los mismos determinantes sociales o medioambientales, lo cual requiere una mayor investigación, pues podrían ser objeto de intervenciones conjuntas.

Como limitaciones, si bien el tamaño muestral es reducido para realizar análisis a profundidad, la cobertura del estudio alcanzó a casi la mitad de la población total existente, siendo esta la primera exploración epidemiológica a las condiciones crónicas de poblaciones Amazónicas del Perú. No se estudiaron marcadores metabólicos ni de inflamación que hubieran complementado el perfil descrito en este estudio; esto fue una decisión de factibilidad en donde intervienen aspectos como control de calidad, particularmente en el almacenamiento y transporte de las muestras, y costos sin sacrificar el objetivo mayor de llegar a estas poblaciones de difícil acceso geográfico. Este estudio no discriminó entre los múltiples usos del cigarrillo (por ejemplo en rituales de curación de medicina tradicional), ni los diferentes aspectos culturales o nutricionales del uso del masato. Sería importante que esto se amplíe con un enfoque cualitativo y participativo.

La discrepancia entre las proporciones de obesidad medidas por BE e IMC podría estar vinculada a que las ecuaciones para la BE no han sido validadas para esta población y no existe un consenso sobre el punto de corte para definir obesidad por BE (11). Se ha reportado que en ciertas poblaciones, sobre todo asiáticas, se encuentra elevados niveles de grasa corporal para bajos valores de IMC; esto es básicamente debido a diferencias que afectan la BE, entre estos (19), la distribución de la grasa corporal, la densidad ósea, las diferencias en la proporción del largo de las piernas. La BE también puede afectarse por el nivel de hidratación y temperatura corporal.

Debido a la logística para la ejecución de este estudio, la presión arterial se midió en una sola visita. Aunque se trató de minimizar el efecto de "bata blanca" tomando la presión más de una vez y con la presencia y traducción de los promotores locales, no es posible descartarlo con certeza, lo cual es relevante en términos de pronóstico y tratamiento ${ }^{(20)}$, pero esto no invalida los resultados desde el punto de vista epidemiológico.

Los hallazgos reportados caracterizan a las comunidades estudiadas y no necesariamente a otras comunidades asháninkas.

En conclusión, si comparamos nuestros resultados con los de otros investigadores, la prevalencia de hipertensión arterial en la población asháninka estudiada, es similar a indígenas que experimentaron migración, aunque mayor a la de pobladores de la selva peruana; sin embargo, la prevalencia de obesidad es menor a ambos grupos. En vista de los cambios a nivel poblacional que ya han ocurrido en otras poblaciones indígenas de la Amazonía ${ }^{(5)}$ y del mundo ${ }^{(4)}$, resulta fundamental la detección y prevención primaria de hipertensión y obesidad, considerando estrategias locales que podrían contribuir a un abordaje culturalmente apropiado de las enfermedades crónicas. Nuestros hallazgos constituyen un llamado para no desatender la situación de las enfermedades crónicas no transmisibles en las poblaciones indígenas de la Amazonia del Perú.

Contribuciones de autoría: CR participó en el diseño, ejecución y análisis e interpretación de los resultados del estudio, y escribió el primer manuscrito. CZ participó activamente en la escritura del manuscrito e interpretación de datos. LC participó en la recolección y obtención de resultados. RHG concibió el estudio y contribuyó en el análisis e interpretación de los resultados. JJM colaboró en el diseño del estudio, aportó en el análisis e interpretación de los resultados, y participó de la escritura del manuscrito. Todos los autores leyeron y aprobaron la versión final del manuscrito.

Fuentes de financiamiento: este estudio fue financiado por el Centro internacional Fogarty del National Institute of Health (NIH) como parte del proyecto "Genetic susceptibility to cancer in Latin America". JJM fue financiado por Wellcome 
Trust (GR074833MA). JJM y RHG como parte de CRONICAS - Centro de Excelencia en Enfermedades Crónicas de la UPCH fueron financiados por el National Heart, Lung and Blood Institute (NHLBI), National Institute of Health (NIH), Department of Health and Human Services, mediante contrato No. HHSN268200900033C.
Conflictos de interés: los autores declaran no tener conflictos de interés.

Descargos de responsabilidad: las opiniones vertidas en este artículo son responsabilidad de los autores y no necesariamente expresa la opinión de sus instituciones.

\section{REFERENCIAS BIBLIOGRÁFICAS}

1. Huicho L, Trelles M, Gonzales F, Mendoza W, Miranda J. Mortality profiles in a country facing epidemiological transition: an analysis of registered data. BMC Public Health. 2009;9:47. doi: 10.1186/1471-2458-9-47.

2. Centro Nacional de Alimentación y Nutricion, Instituto Nacional de Salud. Encuesta Nacional de Indicadores Nutricionales, Bioquímicos, Socioeconómicos y Culturales relacionados con las Enfermedades Crónicas Degenerativas. Lima: Instituto Nacional de Salud; 2006.

3. Dirección General de Epidemiología. Analisis de la situación de los pueblos indígenas de la Amazonía del Perú. Lima: Ministerio de Salud del Perú; 2003.

4. United Nations, Permanent Forum on Indigenous Issues. La situación de los pueblos indígenas del mundo [Internet]. New York: UN; [citado el 5 de octubre del 2013]. Disponible en: http:// www.un.org/esa/socdev/unpfii/documents/SOWIP_fact_sheets_ES.pdf

5. Roriz-Cruz M, Rosset I, Barreto-Roriz R, Mancilha-Carvalho JJ. Acculturation, obesity, and hypertension among female Brazilian Indians. Hypertension. 2010;56(4):e43-4. doi: 10.1161/HYPERTENSIONAHA.110.158956.

6. Balijepalli C, Losch C, Bramlage P, Erbel R, Humphries KH, Jockel KH, et al. Percentile distribution of blood pressure readings in 35683 men and women aged 18 to 99 years. J Hum Hypertens. 2014;28(3):193-200. doi: 10.1038/jhh.2013.85.

7. O'Brien E, Waeber B, Parati G, Staessen J, Myers MG. Blood pressure measuring devices: recommendations of the European Society of Hypertension. BMJ. 2001;322(7285):531-6.
8. RJL Systems. Quantum ii \& Quantum $\mathrm{x}$, Bioelectrical Impedance Analyzers [Internet]. Clinton Township: RJL Systems; 2007 [updated 2007; citado el 24 de noviembre del 2010]. Disponible en: http://www.rjlsystems.com/ products.shtml\#qx

9. National Cholesterol Education Program (NCEP) Expert Panel on Detection, Evaluation, and Treatment of High Blood Cholesterol in Adults (Adult Treatment Panel III). Third Report of the National Cholesterol Education Program (NCEP) Expert Panel on Detection, Evaluation, and Treatment of High Blood Cholesterol in Adults (Adult Treatment Panel III) Final Report. Circulation. 2002;106(25):3143421.

10. World Health Organization (WHO). Obesity and overweight. Fact sheet No311 [Internet]. Geneva: WHO; 2006 [citado el 24 de noviembre del 2010]. Disponible en: http://www. who.int/mediacentre/factsheets/ fs311/en/index.html

11. De Lorenzo A, Deurenberg P, Pietrantuono M, Di Daniele N, Cervelli $\mathrm{V}$, Andreoli A. How fat is obese? Acta Diabetol. 2003;40 Suppl 1(1):S254-7.

12. Mancilha-Carvalho Jde J, Souza e Silva NA. The Yanomami Indians in the INTERSALT Study. Arq Bras Cardiol. 2003;80(3):289-300.

13. Hollenberg NK, Martinez G, McCullough M, Meinking T, Passan D, Preston $M$, et al. Aging, acculturation, salt intake, and hypertension in the Kuna of Panama. Hypertension. 1997;29(1 Pt 2):171-6.

14. Roche ML, Creed-Kanashiro HM, Tuesta I, Kuhnlein HV. Traditional food system provides dietary quality for the Awajún in the Peruvian Amazon. Public Health Nutr. 2008;11(5):457-65.
15. Port Lourenço AE, Ventura Santos R, Orellana JD, Coimbra CE Jr. Nutrition transition in Amazonia: obesity and socioeconomic change in the Suruí Indians from Brazil. Am J Hum Biol. 2008;20(5):564-71. doi: 10.1002/ ajhb.20781.

16. Welch JR, Ferreira AA, Santos RV, Gugelmin SA, Werneck G, Coimbra CE Jr. Nutrition transition, socioeconomic differentiation, and gender among adult Xavante Indians, Brazilian Amazon. Human ecology. 2009;37(1):1326.

17. Bonilla Asalde C. Situación de la tuberculosis en el Perú. Acta Med Peruana. 2008;25(3):163-70.

18. Culqui DR, Trujillo OV, Cueva N, Aylas R, Salaverry O, Bonilla C. Tuberculosis en la población indígena del Perú 2008. Rev Peru Med Exp Salud Publica. 2010;27(1):8-15.

19. Dehghan M, Merchant AT. Is bioelectrical impedance accurate for use in large epidemiological studies? Nutr J. 2008; 7(26):7-26. doi: 10.1186/14752891-7-26.

20. Pickering TG, James GD, Boddie C, Harshfield GA, Blank S, Laragh JH. How common is white coat hypertension? JAMA. 1988;259(2):225-8.

Correspondencia: Candice Romero Dirección: Carlos Gonzales 251, Urbanización Maranga, San Miguel, Lima 32, Perú. Teléfono: (+511) 2090400.

Correo electrónico: romerocandice@gmail.com. 